\title{
Variability in Morphology and Aggressiveness Among North American Vegetative Compatibility Groups of Colletotrichum coccodes
}

\author{
A. M. Aqeel, J. S. Pasche, and N. C. Gudmestad
}

First, second, and third authors: Department of Plant Pathology, North Dakota State University, Fargo 58105. Accepted for publication 30 March 2008.

\begin{abstract}
Aqeel, A. M., Pasche, J. S., and Gudmestad, N. C. 2008. Variability in morphology and aggressiveness among vegetative compatibility groups of Colletotrichum coccodes. Phytopathology 98:901-909.

North American isolates of Colletotrichum coccodes, representing six vegetative compatibility groups (NA-VCG), were used to study morphological and pathogenic variability. The objective was to determine if variability in conidial and microsclerotial size was related to pathogenicity. Significant differences were detected in length, width, and length/width ratios of conidia as well as in the length and width of microsclerotia among the NA-VCGs. The longest and widest conidia were produced by isolates belonging to NA-VCG1 and the largest microsclerotia were produced by isolates of NA-VCG2. Conidial and microsclerotial lengths and widths also were affected significantly by type of growth medium. There was no relationship between the size of conidia and the size of micro-

Aggressiveness of 17 isolates of $C$. coccodes representing six NA-VCG's was studied on three potato cultivars using foliar and root inoculation methods. C. coccodes infection reduced tuber weight in all cultivars with both inoculation methods although tuber weight reductions were significantly higher following root inoculations than foliar inoculations. Pathogenic aggressiveness varied among NA-VCGs. Isolates belonging to NAVCG2 and 3 were the least aggressive on potato foliage and isolates of NA-VCG1, 2, 3, 4, and 5 produced higher microsclerotial density on all three cultivars compared with isolates of NA-VCG6. Across inoculation methods, isolates of $C$. coccodes belonging to NA-VCG2 and 6 were the most aggressive based on reductions in tuber weight. Umatilla Russet was the most susceptible cultivar to $C$. coccodes compared to other cultivars regardless of inoculation method. These results demonstrate variability in morphology and pathogenic aggressiveness among the NA-VCGs of $C$. coccodes but these traits are not related.
\end{abstract} sclerotia among the NA-VCGs studied. Conidial and microsclerotial size may affect inoculum potential and survival as isolates of NA-VCG2 have been demonstrated to occur more frequently than other NA-VCGs.
Additional keywords: black dot, Solanum tuberosum.
Black dot of potato, caused by Colletotrichum coccodes, is both a seed- and soilborne pathogen. The pathogen has been detected in both below- and above-ground plant stems and is able to colonize roots, stolons, tubers, and stems of potato plants (1). The microsclerotia of $C$. coccodes can overwinter in the soil and in infested potato debris for at least 1 year (12) before developing into acervuli on infested debris in the soil, producing conidia that serve as a primary source of inoculum (42). Conidia are disseminated from one plant to another by rain splash or irrigation (9). Infection of potato tubers by $C$. coccodes results in the development of silvery to brown lesions with poorly defined margins on the tuber surface and is characterized by the production of small, black microsclerotia (8). In addition to reducing tuber quality, $C$. coccodes has been demonstrated to reduce yield by as much as $30 \%$ (20).

C. coccodes is an imperfect fungus, with no observed sexual reproduction. Vegetative compatibility is, therefore, the only known means for exchange of genetic material among isolates. Vegetative compatibility groups (VCGs) have been used to study the genetic structure of pathogen populations, identifying formae speciales, races, and aggressiveness groups (24). Vegetative compatibility is genetically regulated, and two isolates are considered in the same group if they anastomose and form stable heterokaryons (24); therefore, isolates belonging to the same VCG have similar pathological and physiological traits $(18,24$, 32,48). The classification of VCGs within a number of plant

Corresponding author: N. C. Gudmestad; E-mail address: neil.gudmestad@ndsu.edu

doi:10.1094/PHYTO-98-8-0901

(c) 2008 The American Phytopathological Society pathogens has been an important step in understanding the genetic structure of the fungus $(16,18,22,24,31,33,38,48)$. For example, VCGs have been useful in understanding the epidemiology of plant pathogens such as Verticillium dahliae and the regional differences that exist within this pathogen population (22). It has been suggested that an increased understanding of the genetic structure of plant pathogens using vegetative compatibility will ultimately assist in improving control of the diseases they cause $(22,31)$.

Nitzan et al. $(31,33)$ used nitrate auxotrophic (Nit) mutants to classify $C$. coccodes isolates, collected from Europe and the Middle East (EU/I), and North America (NA) into four and seven VCGs, respectively. An additional four EU/I VCG's were recently identified (38). Interestingly, no complementation occurs between the NA and EU/I populations, indicating that the $C$. coccodes populations of the two continents are genetically distinct and may have developed independently (33). Amplified fragment length polymorphism (AFLP) primers developed to aid in more rapidly assigning $C$. coccodes isolates to a presumptive NA-VCG correlated well with traditional NA-VCG designations (16). Using AFLP analysis, these investigators were able to study a much larger population of $C$. coccodes in the United States (16). Although they found few differences in the distribution of VCGs of the black dot fungus, there were large differences in the frequency of some groups compared to others. For example, NAVCG4 and 6 were rare while NA-VCG2 and 5 were the most frequently detected (16). Additional information on the phenotypic structure of $C$. coccodes VCGs would improve our understanding of the population biology of this important plant pathogen.

Barkdoll and Davis (2) explored the relationship between $C$. coccodes isolates and pathogenicity to potato utilizing root inocu- 
lations. Variation in the pathogenicity of nine $C$. coccodes isolates was demonstrated on cultivar Russet Burbank, however, these studies were performed prior to the identification of VCGs in this fungus. More recently, differences in aggressiveness were identified among VCGs, also using a root inoculation method (31). Nitzan et al. (31) determined that $C$. coccodes isolates from EU/IVCG3 were more aggressive to potato plants than were other EU/I-VCGs. Later, Nitzan et al. (33) demonstrated that isolates belonging to NA-VCG2 and 5 produced more microsclerotia on the root and plant crown area; however, there were no differences detected in the aggressiveness among NA-VCGs in affecting tuber number or tuber weight in inoculated plants. Differences in pathogenicity were examined among eight $C$. coccodes groups and it was determined that EU/I-VCG3 was the most aggressive to potato based on stem colonization level and sclerotial density on roots and crowns (38). It is apparent from these studies that there is variability in aggressiveness among $C$. coccodes populations but these differences are largely based on microsclerotial development and not yield loss. Furthermore, despite the fact that C. coccodes can infect potato foliage causing significant yield loss (20), no studies have compared the effects of root infections to foliar infections as a method of assessing the aggressiveness of this plant pathogen. Such studies are warranted.

Species of Colletotrichum are identified almost exclusively based on morphological and culture characteristics (39). Characteristics such as conidial morphology, presence or absence of setae, presence or absence of teleomorphs, colony color, production of pigments, and growth rates have been used to differentiate species of Colletotrichum $(3,39)$. Identification of certain Colletotrichum species is difficult due to the extreme variation in morphology, overlapping ranges of conidial morphology, and wide variation in colony characteristics (40). In C. graminicola, two types of conidia have been identified which do not differ in pathogenicity (34). Very little is known regarding the variability in morphology of $C$. coccodes and whether or not differences in conidial and microsclerotial morphology may correlate to pathogenic aggressiveness. Based on our observations, we hypothesize that there are morphological variations among the VCGs and that this variation may correlate with pathogenic aggressiveness. In light of conflicting data on variability in aggressiveness among isolates and VCGs of $C$. coccodes, studies that compare isolate morphology and aggressiveness are warranted. The objectives of this study are to (i) demonstrate the morphological variations of conidia and microsclerotia among the NA-VCGs and to (ii) determine the aggressiveness of NA-VCGs of $C$. coccodes using two inoculation methods (foliar and root).

\section{MATERIALS AND METHODS}

Collection of isolates. $C$. coccodes isolates used in this study originated from potato tubers, roots, stolons, and foliage as well as soil collected from areas where potato production had occurred. Potato tissues were surface-sterilized in $10 \%$ bleach, rinsed thoroughly with distilled water, dried, and plated onto Sorenson's NP-10 semi-selective medium (11). Hyphal-tip or single-spore isolates were produced from each original culture. Cultures were subsequently maintained on $10 \%$ clarified V8 juice (CV8) agar medium (26). For long-term storage, C. coccodes microsclerotia were removed from CV8 agar in a 7.5\% skim milk solution using a sterile bent glass rod, added to silica gel crystals, and stored at $-80^{\circ} \mathrm{C}$ (39). To ensure culture integrity for all trials, frozen stock cultures were plated on agar media prior to each trial. Isolates used is this study were previously assigned to NAVCG $1,2,3,4,5$, or $6(16,33)$.

Conidial morphology. For morphological observation and measurements of $C$. coccodes conidia, 74 isolates representing each NA-VCG (Table 1) were grown on CV8 medium and $1 \%$ potato dextrose agar (PDA) (PDA [10 g/liter], Bacto agar [15 g/liter], and streptomycin sulfate $[0.1 \mathrm{~g} /$ liter $]$ ). The average conidia size of $C$. coccodes isolates was evaluated in six trials, 10 to 15 isolates/trial, and each trial was performed twice with three replications per isolate. In each trial, 1-mm plugs of $C$. coccodes colonized agar were transferred from long term storage onto CV8 and PDA media and incubated for 10 days under continuous fluorescent light at room temperature $\left(25 \pm 2{ }^{\circ} \mathrm{C}\right)$. Four milliliters of sterile distilled water was added to each plate and the agar surface was scraped gently to dislodge the conidia. The suspension was filtered through three layers of autoclaved cheesecloth and the final concentration was adjusted to $10^{4}$ conidia/ml using an hemacytometer. Twenty-five micro-liters of conidial suspension were placed on a sterile glass slide, covered with a sterile cover slip, and stained with cotton blue in a lactophenol solution (100 ml lactophenol, $1 \mathrm{ml}$ of $1 \%$ aqueous cotton blue, and $20 \mathrm{ml}$ glacial acetic acid) (45). To determine conidial length and width, the slides were examined under a compound microscope (Olympus $\mathrm{BX} 40)$ at $\times 100$ magnification. Twenty arbitrarily selected conidia of each $C$. coccodes isolate were measured from each of three prepared slides (replications) for length and width (at the midpoint of the conidia) with the aid of an ocular micrometer calibrated against a fixed known micrometer stage $(2 \mathrm{~mm}$ in length) and divided into 50 units, each unit equal to $0.581 \mu \mathrm{m}$ at $\times 100$.

Microsclerotial morphology. Seventy-four isolates representing each NA-VCG were evaluated for microsclerotial morphology (Table 1). Microsclerotia were produced following a modified procedure described by López-Escudero and Blanco-López (25). In each trial, 1-mm agar plugs colonized with $C$. coccodes were transferred from long-term stock cultures onto CV8 and PDA media and incubated for 14 days under continuous fluorescent light at room temperature $\left(25 \pm 2{ }^{\circ} \mathrm{C}\right)$. Microsclerotia were gently scraped from the agar surface in $4 \mathrm{ml}$ of sterile distilled water. Two milliliters of the microsclerotial suspension and $5 \mu \mathrm{l}$ of lysing enzyme were combined and incubated at $50^{\circ} \mathrm{C}$ for $1 \mathrm{~h}$. Lysing enzyme was prepared as follows: $0.1 \mathrm{~g}$ of lysing enzyme from Trichoderma catalase (Sigma L1412) dissolved in $10 \mathrm{ml}$ of sterile distilled water. To remove all lysed cell debris, the microsclerotial suspension was first filtered through two layers of sterile cheesecloth and the filtrate was retained. The microsclerotial suspension then was captured using Whatman No. 417 $(40 \mu \mathrm{m})$ filter paper, placed in a glass funnel. The filter paper was

TABLE 1. Total number and North American vegetative compatibility group (NA-VCG) for Colletotrichum coccodes isolates used in morphology and aggressiveness evaluations

\begin{tabular}{lclcc}
\hline & \multicolumn{2}{c}{ Morphology } & \multicolumn{2}{c}{ Aggressiveness } \\
\cline { 2 - 3 } \cline { 5 - 6 } NA-VCG & \multicolumn{1}{c}{ Number of isolates } & \multicolumn{1}{c}{ Collection year } & Number of isolates & Collection year \\
\hline NA-VCG1 & 15 & $1989,1990,1995,1997,2002,2003,2004$ & 3 & $1989,1997,2002$ \\
NA-VCG2 & 18 & $1997,2002,2004$ & 3 & 1997,2002 \\
NA-VCG3 & 10 & $1987,1992,1995,1997,2002,2004$ & 2 & $1997,2002,2005$ \\
NA-VCG4 & 2 & 1997,2002 & 3 & 1997,2002 \\
NA-VCG5 & 16 & $1986,1987,1989,1992,2002,2004$ & 3 & 2002,2004 \\
NA-VCG6 & 13 & $1984,1996,1997,2002,2004$ & 3 & 2002 \\
\hline
\end{tabular}

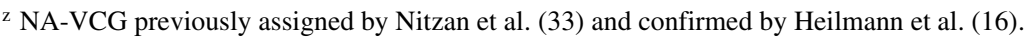


rinsed thoroughly with sterile distilled water and the microsclerotia were washed from the filter paper.

A $35-\mu$ suspension containing at least 10 microsclerotia was placed at the center of a sterile slide, covered with a sterile coverslip, and stained with cotton blue in lactophenol solution. The lengths and widths of 10 microsclerotia from each of three prepared slides (replications) were determined under a compound microscope (Nikon Model Eclipse E400, Japan) at $\times 40$ magnification with the aid of an ocular micrometer. The ocular micrometer calibrated against a fixed and known micrometer stage ( $2 \mathrm{~mm}$ in length). The ocular micrometer divided into 50 units, each unit equal to $4.5 \mu \mathrm{m}$ at $\times 40$.

Plant material. Tuber pieces $(\approx 50 \mathrm{~g})$ obtained from North Dakota certified seed potatoes of three cultivars, Russet Burbank, Umatilla Russet, and Russet Norkotah were cut from surfacesterilized tubers (10\% bleach for $10 \mathrm{~min}$ ), allowed to suberize and grown in a 4-liter pot containing Sunshine potting mixture of peat, vermiculite, and perlite (1:1:1, vol:vol) (Sun-Gro Horticulture Canada, LTD; Seba Beach, Alberta). Potato cultivars Russet Burbank and Russet Norkotah have been used in previous studies involving $C$. coccodes $(20,29,30,33)$ and Umatilla Russet was included here based on field observations by one of the authors (Gudmestad) that it is very susceptible to black dot. Approximately $12 \mathrm{~g}$ of Multicote 6 extra fertilizer 15-15-15 plus micronutrients (Haifa Chemicals, LTD; Haifa Bay, Israel) was added to the soil of each pot after planting. Plants were grown in the greenhouse $\left(20 \pm 5^{\circ} \mathrm{C}, 16 \mathrm{~h}\right.$ light $)$ until an average height of 8 to $10 \mathrm{~cm}$ was reached $(\approx 4$ weeks). Experiments were conducted at similar times of the year (November through March) in a greenhouse at North Dakota State University during 2004, 2005, and 2006.

Preparation of inoculum. Seventeen isolates of $C$. coccodes, collected from infected potato plants from different locations in the United States and Canada (Table 1) were evaluated for aggressiveness. Isolates were chosen based on their assigned VCG and preliminary studies were performed to confirm their pathogenicity. Nine isolates were obtained from the culture collections of J. Miller, University of Idaho and D. Johnson, Washington State University, and eight isolates were used from N. C. Gudmestad's culture collection at North Dakota State University. Fungal isolates were cultured on CV8 agar in 9-cm petri plates for 10 to 14 days at $25^{\circ} \mathrm{C}$ under continuous light. The conidia were harvested with $4 \mathrm{ml}$ of sterile distilled water by scraping the conidia from the agar surface and filtering through three layers of autoclaved cheesecloth. The final concentration of conidial suspensions was adjusted to $10^{5}$ conidia/ml using a hemacytometer. Before inoculation, $35 \mu$ of Tween-20 (Sigma)/100 ml was added to the final conidial suspension. Inocula of each NA-VCG were used to inoculate either roots or foliage of each cultivar in a factorial experiment involving six $C$. coccodes isolate groups $\times$ three cultivars $\times$ two inoculation methods.

Foliar inoculation. The foliar inoculation method used in this study was modified from previously established methods (20). The foliage of 4-week-old plants was wounded with sterile sand blown from a sandblaster (Speedaire Model 6W614, Dayton Electric Mfg. Co., Niles, IL) at a distance of 1.0 to $1.5 \mathrm{~m}$ for $5 \mathrm{~s}$; the air pressure of the sandblaster was $166 \mathrm{kPa}$, creating a wind velocity approximately $14 \mathrm{~km} / \mathrm{h}$ at the plant surface. The foliage was then inoculated with approximately $5 \mathrm{ml}$ conidial suspension of each selected isolate using a hand-held sprayer. Each plant was inoculated separately with an individual isolate. Control plants were sandblasted and inoculated with sterile distilled water. After inoculation, plants were placed in a growth chamber $\left(21^{\circ} \mathrm{C}, 14 \mathrm{~h}\right.$ of light) (Humidifier 1626D, Phytotronics, Inc., Earth City, MO); the mist cycle was $5 \mathrm{~s}$ every $5 \mathrm{~min}$ for $36 \mathrm{~h}$. Plants then were moved into the greenhouse until harvest. Four to six leaves from each plant were collected at harvest to confirm black dot disease symptoms. Leaves were sterilized using $70 \%$ ethanol for $2 \mathrm{~min}$, and then washed with distilled water and placed in 1\% PDA medium with streptomycin. Plates were checked 7 days later for C. coccodes colonization. Percentage severity of foliar infection was recorded weekly for 10 weeks after inoculation. The data from disease severity were used to calculate the relative area under disease progress curve (RAUDPC) using the following formula: RAUDPC $=\left[\sum_{i=1}\left(\left(x_{i+1}+x_{i}\right) / 2\right)\left(t_{i+1}-t_{i}\right)\right] /\left(t_{n}-t_{i}\right)$, where $x_{i}=$ disease severity, $t$ is time in days of each reading and $\mathrm{n}$ is the number of readings (5).

Root inoculation. The root inoculation method used in these studies has been previously described by Nitzan et al. (31). Plants were removed from pots and soil was rinsed from the root system with tap water. Plant roots were immersed in either a conidial suspension $\left(10^{5}\right.$ conidia/ml) or sterile water (control) for $20 \mathrm{~min}$ prior to transplanting into pots filled with a sterile mixture of peat, vermiculite, and perlite $(1: 1: 1, \mathrm{vol} / \mathrm{vol})$. Plants were maintained in the greenhouse until harvest. The tubers and plant root systems were stored at $5^{\circ} \mathrm{C}$ until data collection and sampling. Five segments of roots and stolons (each $2 \mathrm{~cm}$ in length) taken from each plant were surface-sterilized in $10 \%$ bleach for $5 \mathrm{~min}$ and then placed in 1\% PDA with streptomycin to confirm root infection with $C$. coccodes. Microsclerotial density on roots was estimated visually at harvest, 60 days after inoculation, according to the following scale: 0 to 4 (33), where $0=$ no microsclerotia, $1=1$ to $25 \%, 2=26$ to $50 \%, 3=51$ to $75 \%$, and $4=76$ to $100 \%$ of plant tissue colonized by microsclerotia. For both foliar and root inoculated plants, soil gently was removed from the pots and all tubers were collected in paper bags and placed aside for 1 day to allow wound healing before storage at $5^{\circ} \mathrm{C}$. For both inoculation methods, tuber number and weight were recorded at harvest, and converted into percentage of reduction in tuber number and weight compared to the noninoculated controls (17).

Experimental designs and statistical analyses. $C$. coccodes conidial and microsclerotial length, width, and length/width (L/W) ratio were evaluated in six separate trials including 10 to 15 isolates per trial in a completely random design (CRD). Each experiment was performed twice, Levene's method was utilized to test the homogeneity of variance among the independent experiments (27), and data were combined for further analyses. A twofactor analysis of variance (ANOVA) with main affects of NAVCG and medium type was performed using the general linear models procedure (Proc GLM) in Statistical Analysis System (SAS 9.1; SAS Institute Inc., Cary, NC). Differences in mean conidial and microsclerotial length, width, and L/W ratio were compared using Fisher's protected least significant difference (LSD) test $(\alpha=0.05)$. The relationship among and between morphology parameters as well as among and between media types was compared using Pearson's correlation analysis.

The aggressiveness of 17 C. coccodes isolates, representing each of the six NA-VCGs (three isolates from each NA-VCG except NA-VCG4 where only two isolates could be identified), were evaluated in the greenhouse under controlled environmental conditions in a randomized complete block experimental design (RCBD) with two replications. One isolate from each of the six NA-VCGs was used to inoculate plants in each of the three experiments, (no isolate from NA-VCG4 was used in the first experiment); each experiment was performed twice. Using independently and normally distributed error, the percentage reduction means were plotted against the variance to confirm the errors heterogeneity. The percentage reduction data were transformed to $\operatorname{logarithm}(\mathrm{Y}+1 ; \mathrm{Y}$ is the original data) (23), and the transformed data were used for ANOVA and mean comparisons. Levene's test was conducted to test the homogeneity of variance among the independent experiments, and data were combined for further analyses. Transformed percentage reduction of tuber number and weight, RAUDPC, and microsclerotial density on root tissue were analyzed in the general linear models procedure (Proc GLM) in SAS (SAS 9.1; SAS Institute). A three-factor (NA-VCG, cultivar, 
and inoculation method) ANOVA was performed and mean separation was determined using Fisher's protected LSD test $(\alpha=$ $0.05)$. Means and Fisher's protected LSD values were back-transformed for interpretation and presentation of results. The relationship among and between aggressiveness parameters was compared using Pearson's correlation analysis.

\section{RESULTS}

Conidial morphology. Differences were detected in conidial morphology among isolates of six NA-VCGs of $C$. coccodes grown on two types of media (PDA and CV8). A significant NAVCG $\times$ medium interaction was observed for all parameters of conidia length, width, and L/W ratios caused primarily by the differential response of isolates belonging to NA-VCG4 on PDA vs. CV8. There were significant differences within NA-VCGs and medium type for conidia length, width, and length/width ratio (Table 2). When comparing conidial size among NA-VCGs and across media types, conidia produced from isolates belonging to NA-VCG1 were significantly longer $(16.43 \mu \mathrm{m})$ than conidia from isolates of any other NA-VCG $(P<0.0001)$ and also significantly wider $(3.41 \mu \mathrm{m})$ than isolates from all NA-VCGs except isolates of NA-VCG4 $(P=0.002)$ (Table 2). While isolates belonging to NA-VCG2 produced the shortest conidia $(12.75 \mu \mathrm{m})$ this difference was only significant when compared to isolates of NA-VCG1 and 6. There were few differences in the length of conidia among isolates belonging to NA-VCG 3, 4, and 6. C. coccodes isolates belonging to NA-VCG5 produced the narrowest conidia $(2.99 \mu \mathrm{m})$, significantly narrower than conidia produced by isolates belonging to NA-VCG1. These differences are further demonstrated by significantly higher conidial L/W ratios from conidia produced from isolates belonging to NA-VCG1 (4.83) $(P<0.0001)$ which tended to be more elongated compared with those from isolates from all other NA-VCGs (Table 2). Based on conidial $\mathrm{L} / \mathrm{W}$ ratios, the $C$. coccodes isolates can be classified into two groups: (i) L/W ratio less than 4.3 in isolates of NA-VCG2 and 4 ; and (ii) L/W ratio greater than 4.3 in isolates of NA-VCG1, 3,5 , and 6 .

For each of the isolates evaluated, conidial dimensions were compared when grown on CV8 and PDA. Across all NA-VCGs, C. coccodes conidia produced on CV8 media were significantly larger and more elongated than the comparably smaller and more rounded conidia produced on PDA (Table 2) as demonstrated by comparing $\mathrm{L} / \mathrm{W}$ ratios for each media type $(P<0.0001)$. Conidial length and width of $C$. coccodes isolates grown on a single medium, either CV8 $\left(R^{2}=0.76\right)$ or PDA $\left(R^{2}=0.77\right)$, were linearly correlated indicating that on a single medium as conidia lengthened they also became wider, although length tended to be more sensitive to and more variable on PDA media compared to width. Also, there was a strong correlation between conidial length on CV8 versus length on PDA $\left(R^{2}=0.80\right)$, and conidial width on CV8 versus width on PDA $\left(R^{2}=0.75\right)$.

Microsclerotial morphology. Similar to conidial morphology experiments, significant differences existed in microsclerotial morphology among six NA-VCGs of $C$. coccodes grown on CV8 and PDA. A significant NA-VCG $\times$ medium interaction was observed for the parameters of microsclerotial length and width caused primarily by the differential response of isolates belonging to NA-VCG6 when grown on PDA versus CV8 relative to isolates belonging to other groups. There were also significant differences among NA-VCGs and between medium types for microsclerotial length and width (Table 3). No significant differences were observed for microsclerotial L/W ratios (data not shown).

Across media types, microsclerotia produced by isolates belonging to NA-VCG2 were significantly longer $(185.31 \mu \mathrm{m})(P<$ $0.0001)$ and wider $(167.03 \mu \mathrm{m})(P<.0001)$ than those produced by isolates belonging to any other NA-VCGs while microsclerotia produced by isolates belonging to NA-VCG5 were significantly

TABLE 2. Conidial length and width $(\mu \mathrm{m})$ of Colletotrichum coccodes isolates from six North American vegetative compatibility groups (NA-VCG) grown on potato dextrose agar (PDA) and 10\% clarified V8 juice agar (CV8)

\begin{tabular}{|c|c|c|c|c|c|c|c|c|c|}
\hline \multirow[b]{3}{*}{ NA-VCG } & \multicolumn{3}{|c|}{ Conidial length $^{\mathrm{z}}(\mu \mathrm{m})$} & \multicolumn{3}{|c|}{ Conidial width ${ }^{\mathrm{z}}(\mu \mathrm{m})$} & \multicolumn{3}{|c|}{ Conidial length/width ratio } \\
\hline & \multicolumn{2}{|c|}{ Media type } & \multirow[b]{2}{*}{ Mean } & \multicolumn{2}{|c|}{ Media type } & \multirow[b]{2}{*}{ Mean } & \multicolumn{2}{|c|}{ Media type } & \multirow[b]{2}{*}{ Mean } \\
\hline & CV8 & PDA & & CV8 & PDA & & CV8 & PDA & \\
\hline NA-VCG1 & $17.02 \mathrm{a}$ & $15.48 \mathrm{a}$ & $16.43 \mathrm{a}$ & $3.42 \mathrm{a}$ & $3.38 \mathrm{a}$ & $3.41 \mathrm{a}$ & $4.98 \mathrm{ab}$ & $4.58 \mathrm{a}$ & $4.83 \mathrm{a}$ \\
\hline NA-VCG2 & $14.40 \mathrm{c}$ & $10.53 \mathrm{~d}$ & $12.75 \mathrm{c}$ & $3.07 \mathrm{~b}$ & $2.99 \mathrm{~b}$ & $3.03 \mathrm{~b}$ & $4.71 \mathrm{ab}$ & $3.59 \mathrm{c}$ & $4.24 \mathrm{c}$ \\
\hline NA-VCG3 & $15.05 \mathrm{bc}$ & $11.01 \mathrm{~cd}$ & $13.36 \mathrm{bc}$ & $3.24 \mathrm{ab}$ & $2.79 \mathrm{~b}$ & $3.05 \mathrm{~b}$ & $4.65 \mathrm{~b}$ & $3.96 \mathrm{~b}$ & $4.37 \mathrm{bc}$ \\
\hline NA-VCG4 & $15.10 \mathrm{bc}$ & $12.19 \mathrm{~b}$ & $13.65 \mathrm{bc}$ & $3.12 \mathrm{ab}$ & $3.53 \mathrm{a}$ & $3.32 \mathrm{ab}$ & $4.83 \mathrm{ab}$ & $3.46 \mathrm{c}$ & $4.15 \mathrm{c}$ \\
\hline NA-VCG5 & $15.13 \mathrm{bc}$ & $11.44 \mathrm{c}$ & $13.31 \mathrm{bc}$ & $3.16 \mathrm{ab}$ & $2.83 \mathrm{~b}$ & $2.99 \mathrm{~b}$ & $4.86 \mathrm{ab}$ & $4.08 \mathrm{~b}$ & $4.48 \mathrm{~b}$ \\
\hline NA-VCG6 & $16.21 \mathrm{ab}$ & $11.12 \mathrm{~cd}$ & $14.03 \mathrm{~b}$ & $3.19 \mathrm{ab}$ & $2.85 \mathrm{~b}$ & $3.04 \mathrm{~b}$ & $5.08 \mathrm{a}$ & $3.91 \mathrm{~b}$ & $4.58 \mathrm{~b}$ \\
\hline Mean & $15.57 \mathrm{a}$ & $11.85 \mathrm{~b}$ & & $3.21 \mathrm{a}$ & $2.97 \mathrm{~b}$ & & $4.86 \mathrm{a}$ & $4.01 \mathrm{~b}$ & \\
\hline
\end{tabular}

${ }^{\mathrm{z}}$ Conidial length and width based on an average of 60 conidia per isolate for each media type. Fisher's protected least significant difference (LSD) value ( $\alpha=$ 0.05 ) for the interaction between NA-VCG and media type equals 1.37 and 0.28 for conidial length and width, respectively. Mean separation based upon Fisher's protected LSD test $(\alpha=0.05)$. Means within columns followed by the same letter are not significantly different.

TABLE 3. Microsclerotial length and width $(\mu \mathrm{m})$ of Colletotrichum coccodes isolates from six North American vegetative compatibility groups (NA-VCG) grown on potato dextrose agar (PDA) and 10\% clarified V8 juice agar (CV8)

\begin{tabular}{|c|c|c|c|c|c|c|}
\hline \multirow[b]{3}{*}{ NA-VCG } & \multicolumn{3}{|c|}{ Microsclerotia length $^{\mathrm{z}}(\mu \mathrm{m})$} & \multicolumn{3}{|c|}{ Microsclerotia width $^{\mathrm{z}}(\mu \mathrm{m})$} \\
\hline & \multicolumn{2}{|c|}{ Media type } & \multirow[b]{2}{*}{ Mean } & \multicolumn{2}{|c|}{ Media type } & \multirow[b]{2}{*}{ Mean } \\
\hline & CV8 & PDA & & CV8 & PDA & \\
\hline NA-VCG1 & $177.33 \mathrm{c}$ & $134.06 \mathrm{~d}$ & $155.46 \mathrm{c}$ & $157.24 \mathrm{c}$ & $122.56 \mathrm{c}$ & $139.71 \mathrm{c}$ \\
\hline NA-VCG2 & $192.46 \mathrm{ab}$ & $174.16 \mathrm{a}$ & $185.31 \mathrm{a}$ & $178.68 \mathrm{ab}$ & $155.39 \mathrm{a}$ & $167.03 \mathrm{a}$ \\
\hline NA-VCG3 & $154.16 \mathrm{~d}$ & $120.66 \mathrm{e}$ & $137.41 \mathrm{~d}$ & $143.22 \mathrm{~d}$ & $107.96 \mathrm{~d}$ & $125.59 \mathrm{~d}$ \\
\hline NA-VCG4 & $183.28 \mathrm{bc}$ & $151.48 \mathrm{~b}$ & $167.38 \mathrm{~b}$ & $176.05 \mathrm{~b}$ & $142.25 \mathrm{~b}$ & $159.15 \mathrm{~b}$ \\
\hline NA-VCG5 & $133.90 \mathrm{e}$ & $102.50 \mathrm{f}$ & $118.20 \mathrm{e}$ & $125.05 \mathrm{e}$ & $99.21 \mathrm{e}$ & $112.13 \mathrm{e}$ \\
\hline NA-VCG6 & $193.86 \mathrm{a}$ & $142.30 \mathrm{c}$ & $168.08 \mathrm{~b}$ & $188.62 \mathrm{a}$ & $127.30 \mathrm{c}$ & $157.96 \mathrm{~b}$ \\
\hline Mean & $171.46 \mathrm{a}$ & $135.75 \mathrm{~b}$ & & $159.97 \mathrm{a}$ & $123.68 \mathrm{~b}$ & \\
\hline
\end{tabular}

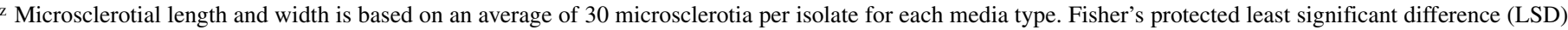
value $(\alpha=0.05)$ for the interaction between NAVCG and media type equals 10.50 and 11.50 for microsclerotial length and width, respectively. Mean separation based upon Fisher's protected LSD test $(\alpha=0.05)$. Means within columns followed by the same letter are not significantly different. 
shorter $(118.2 \mu \mathrm{m})$ and narrower $(112.13 \mu \mathrm{m})$ than those produced by isolates of any other NA-VCG (Table 3). Across media types, no correlation existed between conidial and microsclerotial length $\left(R^{2}=0.015\right)$ or width $\left(R^{2}=0.001\right)$ or $\mathrm{L} / \mathrm{W}$ ratio $\left(R^{2}=0.001\right)$.

Among NA-VCGs, both microsclerotial lengths and widths were significantly smaller when grown on PDA $(135.75 \mu \mathrm{m}$ long $\times$ $123.68 \mu \mathrm{m}$ wide $)(P<.0001)$ compared to CV8 $(171.46 \mu \mathrm{m}$ long $\times$ $159.97 \mu \mathrm{m}$ wide $)(P<.0001)$ (Table 3$)$, similar to results observed for conidial morphology. Microsclerotial length and width of $C$. coccodes isolates grown on a single medium, either CV8 $\left(R^{2}=\right.$ $0.89)$ or PDA $\left(R^{2}=0.92\right)$, were linearly correlated but length to width ratios, CV8 versus PDA $\left(R^{2}=0.003\right)$ were not. Additionally, there was a correlation between microsclerotial length on CV8 versus length on PDA $\left(R^{2}=0.58\right)$, and microsclerotial width on CV8 versus width on PDA $\left(R^{2}=0.44\right)$. Based on the morphological parameter of length, microsclerotia can be classified into four groups: (i) length greater than $185 \mu \mathrm{m}$ in isolates belonging to NA-VCG2, (ii) length between 150 to $180 \mu \mathrm{m}$ in isolates of NA-VCG1, 4, and 6, (iii) length between 130 to 150 $\mu \mathrm{m}$ in isolates of NA-VCG3, and (iv) length less than $130 \mu \mathrm{m}$ in isolates of NA-VCG5.

Aggressiveness studies. Significant differences in percentage reduction of tuber weight were observed for all three main effects of potato cultivar, NA-VCG, and method of inoculation. There was also a significant interaction in percentage reduction of tuber weight for cultivar $\times$ inoculation method but not NA-VCG $\times$ inoculation method, cultivar $\times$ NA-VCG or cultivar $\times$ inoculation method $\times$ NA-VCG (Tables 4 and 5). Russet Burbank was the least susceptible cultivar to foliar infections of $C$. coccodes but the most susceptible to root infections (Table 4) which contributed to the significant cultivar $\times$ inoculation method interaction. Across NA-VCG's and inoculation methods, Umatilla Russet was the most susceptible cultivar to C. coccodes, displaying tuber weight reductions of $63.6 \%$ compared with noninoculated controls $(P=0.019)$ (Table 4). Tuber weights across cultivars and NA-VCGs were reduced at a significantly higher percentage with root inoculations versus foliar inoculations (Tables 4 and 5). Across inoculation methods and cultivars, isolates belonging to NA-VCG2 and 6 were significantly more aggressive, resulting in the greatest reduction in tuber weight $(P=$ 0.039 ) and isolates belonging to NA-VCG3 and 4 were the least aggressive. Isolates of $C$. coccodes belong to NA-VCG3, 4 and 5 caused significantly less reduction in tuber weight when inoculated onto foliage $(P=0.013)$, however, there were no differences among NA-VCGs in tuber weight reductions following root inoculation (Table 5). No significant differences occurred in per-

TABLE 4. Mean percentage reduction in tuber weight of three cultivars inoculated with Colletotrichum coccodes by two inoculation methods

\begin{tabular}{|c|c|c|c|}
\hline \multirow[b]{3}{*}{ Cultivar } & \multicolumn{3}{|c|}{$\%$ Tuber weight reduction ${ }^{\mathrm{x}}$} \\
\hline & \multicolumn{2}{|c|}{ Inoculation method } & \multirow[b]{2}{*}{ Mean } \\
\hline & Foliar $^{\mathrm{y}}$ & $\operatorname{Root}^{\mathrm{z}}$ & \\
\hline Russet Burbank & $52.7 \mathrm{~b}$ & $66.3 \mathrm{a}$ & $59.2 \mathrm{~b}$ \\
\hline Russet Norkotah & $55.9 \mathrm{~b}$ & $60.3 \mathrm{~b}$ & $58.1 \mathrm{~b}$ \\
\hline Umatilla Russet & $61.4 \mathrm{a}$ & $65.6 \mathrm{a}$ & $63.6 \mathrm{a}$ \\
\hline Mean & $56.7 \mathrm{~b}$ & $64.1 \mathrm{a}$ & \\
\hline
\end{tabular}

${ }^{x}$ Percentage reduction of tuber weight (PR) calculated using the following formula: $\mathrm{PR}=([\mathrm{A}-\mathrm{B} / \mathrm{B}] \times-100)$, where $\mathrm{A}$ is treatment's tuber weight or number, and $\mathrm{B}$ is nontreated control tuber weight or number. Data were transformed prior analysis using the $\operatorname{logarithm}(\mathrm{Y}+1$; $\mathrm{Y}$ is the original data); the means and the Fisher's protected least significant difference (LSD) values were back-transformed for interpretation and presentation.

${ }^{y}$ Conidia were applied to foliage of each cultivar previously wounded via sandblasting.

${ }^{\mathrm{z}}$ Roots were dipped into a conidial suspension. Fisher's protected LSD value $(\alpha=0.05)$ for interaction between cultivar and method equals 3.5. Mean separation based upon Fisher's protected LSD test $(\alpha=0.05)$. Means within columns followed by the same letter are not significantly different. centage reduction of tuber number among the main effects of cultivar, NA-VCG and inoculation methods (data not shown).

Significant differences in foliar disease development based on RAUDPC were detected among cultivars and NA-VCGs. Similar to results from tuber weight, Umatilla Russet was significantly more susceptible to foliar infection by $C$. coccodes followed by Russet Norkotah and Russet Burbank $(P<0.0001)$ (Fig. 1A). Isolates of $C$. coccodes belonging to NA-VCG2 and 3 were significantly less aggressive by foliar infection than the other groups $(P<0.0001)$ (Fig. 1B). A significant cultivar $\times$ NA-VCG interaction also existed. The response trend of these three cultivars to inoculation with isolates of NA-VCG2, 3, 5, and 6 was similar, with Umatilla Russet susceptible, and Russet Norkotah as moderately susceptible, and Russet Burbank the least susceptible (data not shown). However, cultivar responses varied when inoculated with isolates of $C$. coccodes belonging to NA-VCG1 and 4. In Russet Burbank and Umatilla Russet, isolates of NAVCG1, 5, and 6 caused more foliar damage while in Russet Norkotah, isolates belonging to NA-VCG4, 5, and 6 caused more foliar damage than did isolates of NA-VCG1 (data not shown). No correlation existed $\left(R^{2}=0.003\right)$ between tuber weight reduction in foliar inoculated plants and RAUDPC mainly due to the differing reactions of isolates of NA-VCG2 and 4. Isolates belonging to all other NA-VCGs tended to give relatively similar responses.

Significant differences were detected in microsclerotial density on roots among cultivars and NA-VCGs, but no interaction was observed between cultivar and NA-VCG. Microsclerotial density was significantly higher on roots of Umatilla Russet versus Russet Norkotah $(P=0.024)$ although not significantly higher than Russet Burbank (Fig. 2A). Plant roots inoculated with isolates belonging to NA-VCG6 had significantly lower $(P=$ 0.013 ) microsclerotial densities on roots when compared to isolates of other NA-VCG's (Fig. 2B). As was the case with RAUDPC, microsclerotial density was not correlated with tuber weight reductions $\left(R^{2}=0.008\right)$. This was mainly due to isolates of NA-VCG6, which caused the most reduction in tuber weight, but had significantly lower microsclerotial density.

\section{DISCUSSION}

There are few studies on the morphology of conidia and microsclerotia of $C$. coccodes and those that exist were published

TABLE 5. Mean percentage reduction in tuber weight in potatoes inoculated with six North American vegetative compatibility groups (NA-VCG) of Colletotrichum coccodes by two inoculation methods

\begin{tabular}{lccc}
\hline & \multicolumn{3}{c}{$\%$ Reduction in tuber weight $^{\mathrm{x}}$} \\
\cline { 2 - 3 } NA-VCG & \multicolumn{2}{c}{ Inoculation method } & Mean \\
\cline { 2 - 3 } NA-VCG1 & $58.8 \mathrm{a}$ & Root $^{\mathrm{z}}$ & $60.7 \mathrm{ab}$ \\
NA-VCG2 & $62.9 \mathrm{a}$ & $62.5 \mathrm{a}$ & $63.7 \mathrm{a}$ \\
NA-VCG3 & $51.0 \mathrm{~b}$ & $64.4 \mathrm{a}$ & $57.0 \mathrm{c}$ \\
NA-VCG4 & $52.4 \mathrm{~b}$ & $62.9 \mathrm{a}$ & $57.0 \mathrm{c}$ \\
NA-VCG5 & $52.7 \mathrm{~b}$ & $62.2 \mathrm{a}$ & $60.3 \mathrm{bc}$ \\
NA-VCG6 & $62.9 \mathrm{a}$ & $68.3 \mathrm{a}$ & $64.1 \mathrm{a}$ \\
Mean & $56.7 \mathrm{a}$ & $64.8 \mathrm{a}$ & \\
\hline
\end{tabular}

${ }^{x}$ Percentage reduction of tuber weight $(\mathrm{PR})$ calculated using the following formula: $\mathrm{PR}=[(\mathrm{A}-\mathrm{B} / \mathrm{B}) \times-100]$, where $\mathrm{A}$ is treatment's tuber weight or number, and $\mathrm{B}$ is nontreated control tuber weight or number. Data were transformed prior analysis using the $\operatorname{logarithm}(\mathrm{Y}+1$; $\mathrm{Y}$ is the original data); the means and the Fisher's protected least significant difference (LSD) values were back-transformed for interpretation and presentation.

y Conidia were applied to foliage of each cultivar previously wounded via sandblasting.

${ }^{\mathrm{z}}$ Roots were dipped into a conidial suspension. The interaction between NAVCG and method was not significant. Mean separation based upon Fisher's protected LSD test $(\alpha=0.05)$. Means within columns followed by the same letter are not significantly different. 
before the identification of VCGs (39). During the course of previous studies on molecular genetic variability in $C$. coccodes (16), it became apparent that there were significant differences in conidial and microsclerotial morphology among NA-VCGs. Since there are known differences in pathogenic aggressiveness among EU/I- and NA-VCGs of $C$. coccodes, it appeared that studies potentially relating these two observations would be worthwhile. The objective of this study was to distinguish conidial and microsclerotial morphology among $C$. coccodes isolates belonging to six NA-VCGs and to determine if any differences may be reflected in pathogenic aggressiveness. Additionally, since a previous study found few differences in aggressiveness among NAVCGs using a root inoculation method (33), and since $C$. coccodes is a known foliar pathogen (20), it also seemed important to evaluate aggressiveness among the VCGs using more than one inoculation method. The differences detected among the VCGs evaluated add significantly to our knowledge of the $C$. coccodes population in North America.

Conidial lengths of $C$. coccodes isolates examined in the current research were very similar to other Colletotrichum species, such as $C$. dematium, C. acutatum, C. nupharicola, and $C$. nymphaeae $(21,36)$. Microsclerotia form abundantly and are black, setose, globose to oval with setae, and arranged in a series of circles around the center of transferred colony plug. The morphology of conidia and microsclerotia are useful characters for discriminating species, despite an overlap in length and width with other Colletotrichum species (40). The studies reported here demonstrated the size of conidia and microsclerotia varied sig-
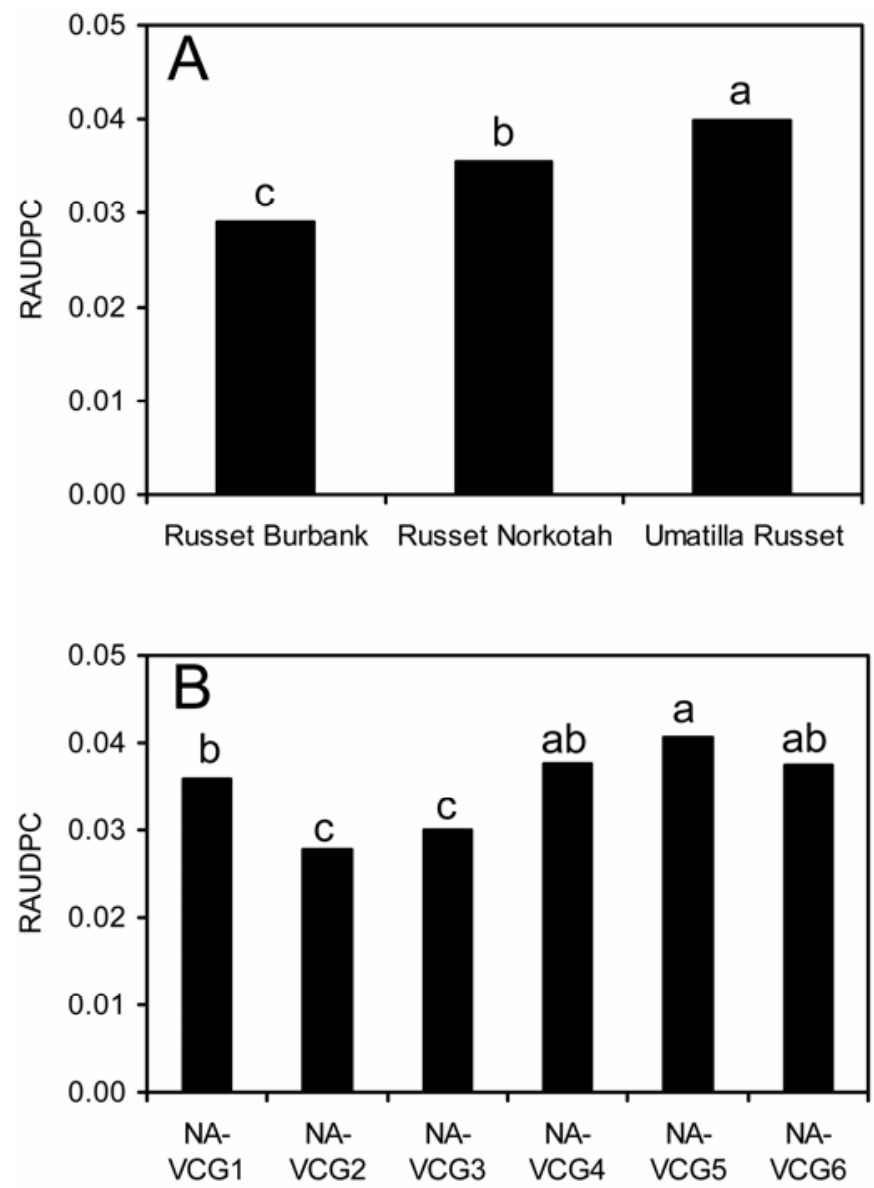

Fig. 1. Comparison of mean relative area under disease progress curve (RAUDPC) among A, three potato cultivars and B, six North American vegetative compatibility groups (NA-VCG) following foliar inoculation of cultivars with Colletotrichum coccodes. Mean separation based upon Fisher's protected least significant difference test $(\alpha=0.05)$. Bars denoted by the same letter are not significantly different. nificantly among VCGs of $C$. coccodes. Variability in conidial and microsclerotial size among VCGs has been reported previously in Verticillium dahliae $(22,48)$. The findings in the present study further support the hypothesis that VCGs of $C$. coccodes differ in physiological and morphological traits and it is not surprising to find this variation since the variability in physiological traits of $C$. coccodes have been previously related to the VCG $(32,38)$. The basis for morphological variations among NA-VCGs is not known at this time but may be related to variations in ecological adaptation within NA $C$. coccodes populations.

Culture media influenced the size and shape of conidia as well as size of microsclerotia. Conidia and microsclerotia harvested from CV8 were greater in length and width. This variation is likely due to differences in the nutritional components of the two media. According to the CV8 juice label, it is a juice made from tomatoes and several other vegetables containing carbohydrate, sodium, potassium, sugar, and protein (Campbell's Soup Company, Camden, NJ). PDA is a general-purpose medium for yeasts and many pathogenic fungi. The main components of PDA are potato starch and dextrose, which support luxuriant fungal growth. Sucrose and calcium have been reported as important elements for $C$. coccodes vegetative growth and sporulation (46). Although the effects of different types of media on $C$. coccodes conidial size were evaluated in this study, the balance of the nutrients, especially the carbon-to-nitrogen ratio, may also affect fungal growth. Yu et al. (47) found that growth and sporulation of C. coccodes increased with increasing C:N ratio from 5:1 to 10:1, and decreased as the $\mathrm{C}: \mathrm{N}$ ratio rose above 15:1. Unfortunately,
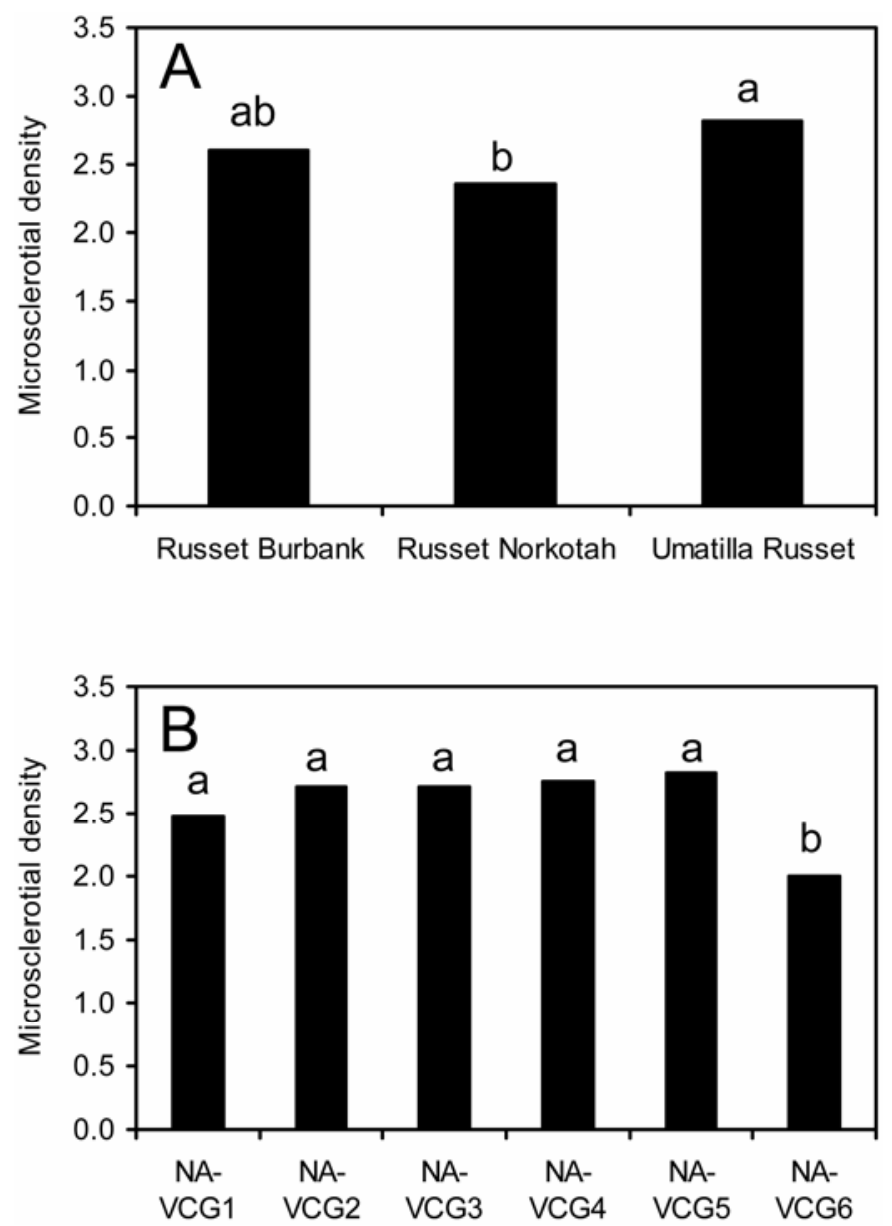

Fig. 2. Comparison of mean microsclerotial density among $\mathbf{A}$, three potato cultivars and B, six North American vegetative compatibility groups (NAVCG) following root inoculation of cultivars with Colletotrichum coccodes. Mean separation based upon Fisher's protected least significant difference test $(\alpha=0.05)$. Bars denoted by the same letter are not significantly different. 
differences in nutrients such as calcium or $\mathrm{C}: \mathrm{N}$ ratios between CV8 and PDA media are not known. López-Escudero and Blanco-López (25) found variation in microsclerotial size of $V$. dahliae isolates grown on two different media (modified sodium polypectate agar and modified sodium polypectate agar with cellophane). The NA-VCG $\times$ medium interaction indicated that the response of each NA-VCG on the media were different and may be due also to physiological variation that exists among VCGs (32). Mordue (28) reported that the conidial size of $C$. coccodes grown on PDA as 16 to $24 \times 2.5$ to $4.5 \mu \mathrm{m}$. In the present study, conidia length and width of $C$. coccodes, regardless of media, ranged from 10.53 to $17.02 \mu \mathrm{m}$ and 2.79 to $3.53 \mu \mathrm{m}$, respectively, a much narrower range than previously reported. Mordue's description (28) did not include microsclerotial length or width parameters.

Previous studies have demonstrated that both root $(2,29,33,41)$ and foliar $(7,20,41)$ infections of $C$. coccodes cause yield loss. However, these previous studies did not directly compare root and foliar infections of this pathogen in the same controlled experiments. In studies reported here, black dot reduced tuber weight, although significantly more so with root inoculations than with foliar inoculations, supporting previous research suggesting that roots are the most sensitive organ to $C$. coccodes infection (1). Tsror (Lahkim) et al. (41) suggested that soil and tuber inoculations with $C$. coccodes result in greater yield reduction than foliar inoculations. However, it is important to note that in the aforementioned study, soil/tuber inoculations and foliar inoculations were not performed in the same experiment so no direct comparisons could be made. It has been reported also that $C$. coccodes grows faster toward the susceptible organs, roots, stolons, and tubers from a stem infection, which appears to confirm the susceptibility of roots to black dot (30). The studies reported here are the first to directly compare foliar and root infections of $C$. coccodes and clearly demonstrate that below ground infections of this pathogen are more economically damaging than above ground infections.

In order to examine the relationship between NA-VCG and the extent of aggressiveness in $C$. coccodes, three cultivars were inoculated with isolates representing six NA-VCGs. Isolates of NA-VCG2 and 6 were the most aggressive resulting in the highest percentage reductions in tuber weight across inoculation methods. Isolates belonging to NA-VCG3 and 4 were the least aggressive. Nitzan et al. (33) determined that isolates of NA-VCGs 1, 2, and 5 caused more yield reduction than isolates of other NA-VCGs, however, these yield reductions were not significantly different among the NA-VCGs studied. In that study (33), the authors concluded that NA-VCG2 and 5 were significantly more aggressive than the other VCGs studied, however, that conclusion was derived primarily due to higher sclerotial densities formed by isolates belonging to these two groups. NA-VCG2 has been reported also to cause a higher disease incidence on daughter tubers (33). Results from these two studies appear to agree that isolates of $C$. coccodes belonging to NA-VCG2 are among the most aggressive, and isolates belonging to NA-VCG3 are among the least aggressive. Disagreement in our results are probably due to differences in inoculation methods since Nitzan et al. (33) used only root inoculations to evaluate aggressiveness while we used a combination of root and foliar inoculations. Interestingly both studies could find no differences in tuber weight reduction among NAVCGs when using a root inoculation method. Therefore, tuber weight reductions as a measurement are insufficient alone to differentiate pathogenic aggressiveness of $C$. coccodes VCGs. This is, perhaps, further indication that roots are the most susceptible organ to black dot on the potato plant, so susceptible that they are insufficient to detect differences in isolate aggressiveness. It should be noted that variation in aggressiveness among VCGs has been reported previously in European/Mediterranean populations of $C$. coccodes using a root inoculation method (31). However, in the results of studies reported here and elsewhere (33), differences in aggressiveness in isolates of $C$. coccodes belonging to different NA-VCGs could only be differentiated by evaluating microsclerotial formation on roots and/or stems following a root inoculation. In contrast, in the studies reported here variability in the aggressiveness of North American $C$. coccodes populations was demonstrated using a foliar inoculation method. Foliar inoculations with isolates belonging to different NA-VCG of C. coccodes resulted in significantly different levels of foliar disease development and in statistically significant reductions in tuber weight. Unfortunately, these two measurements of isolate aggressiveness do not agree.

Umatilla Russet, a late-maturing cultivar, was the most susceptible cultivar to $C$. coccodes infection, which reduced tuber weight in this cultivar up to $63.6 \%$ followed by Russet Burbank, a moderately late-maturing cultivar, and Russet Norkotah, an earlymaturing cultivar. This finding is consistent with the results of the RAUDPC and microsclerotial formation on the same cultivar following foliar and root method of inoculations, respectively, confirming that Umatilla Russet is the most susceptible to black dot of the three cultivars studied. Similar findings were reported by Nitzan et al. (30), in which they found Russet Norkotah less susceptible to $C$. coccodes than Russet Burbank. Differences in cultivar maturity may explain the susceptibility of Umatilla Russet to $C$. coccodes. Umatilla Russet is likely subjected to $C$. coccodes infections for a longer period of time than Russet Norkotah, through continuous $C$. coccodes growth and colonization. Similarly, Davis et al. (7) observed differences among cultivars in susceptibility to $C$. coccodes, with Russet Burbank being the most susceptible and Norgold Russet, an early maturing cultivar, the least. Similar questions are raised in the studies reported here and have been raised with other diseases such as early blight $(6,10,19,35)$. Johanson and Thurston (19) and Pelletier and Fry (35) reported that resistance to early blight is associated with plant age. Early maturing cultivars were more susceptible to early blight while late maturing cultivars were more resistant. The differentiation between early- and late-maturing potato cultivars in susceptibility to plant pathogens is obviously challenging. Several researchers $(6,10,19,35)$ have evaluated and characterized reactions of potato cultivars to early blight disease, based on the components of resistance rather than yield, including incubation period, lesion number and expansion rate, AUDPC, disease severity, and spore production per lesion. Similarly, in the studies reported here it was attempted to use RAUDPC and microsclerotial density on roots to separate susceptibility response among cultivars. Positive correlations have been found between pathogen sclerotial density on roots and plant susceptibility in different crops $(4,41)$. In potato, Nitzan et al. $(31,33)$ used microsclerotial density on roots as a method to determine isolate aggressiveness. In the present study, microsclerotial density was highest on roots of Umatilla Russet. Based on microsclerotial density, isolates belonging to NA-VCGs 2, 3, 4, and 5 isolates were the most aggressive. We have concerns, however, in using semi-quantitative parameters such as microsclerotial density on roots using a rating scale as a method for differentiating isolate aggressiveness or cultivar susceptibility to black dot. Given the variability in microsclerotial sizes among NA-VCGs, sclerotial densities may not be appropriate and we note specifically that NA-VCG2 has been consistently evaluated as the most aggressive isolate group and it also forms the largest microsclerotia based on the studies reported here. We cannot dismiss the fact that we, and others $(31,33)$, may have created an artifact by using microsclerotial density as an assessment method without accounting for differences among populations in the size of these structures. Unfortunately, using percentage yield reduction to differentiate among cultivars may also not be sufficient to assess germplasm susceptibility. This association should be investigated further by using molecular markers closely linked to resistance gene(s) and matur- 
ity gene(s) for efficient selection and identification of resistant potato genotypes as has been done elsewhere in potato $(13,14$, $37,43,44)$.

The biological significance of conidial and microsclerotial size is not immediately evident. Our hypothesis was that isolates of VCGs of $C$. coccodes producing the largest conidia or microsclerotia would be the most aggressive pathogenically, but this does not appear to be true. C. coccodes isolates belonging to NAVCG1 produced the largest conidia and isolates of NA-VCG2 produced the largest microsclerotia. If conidial and microsclerotial size had positively influenced pathogenic aggressiveness, than isolates of $C$. coccodes belonging to these two NA-VCGs would have been the most aggressive causing the greatest reduction in tuber weight, but that was not the case. Across inoculation methods and cultivars, isolates of NA-VCG2 were the second most aggressive; however, these isolates were no more aggressive than isolates belonging to NA-VCG5, which produced the smallest microsclerotia. Interestingly, isolates belonging to NA-VCG2 produce the smallest conidia and are one of the least effective at infecting potato foliage, possibly indicating that conidial size affects survivability. However, since isolates of NA-VCG2 produced the largest microsclerotia and the smallest conidia, isolates belonging to this group may have higher inoculum potential in nature and thus, a selection advantage. An analysis of the $C$. coccodes population (16) found NA-VCG2 to be the most frequently detected group, supporting this hypothesis. The larger microsclerotia of NA-VCG2 may survive longer than the smaller microsclerotia of other VCGs, as is the case with Sclerotinia sclerotiorum (15). Further investigation is necessary to determine if differences in the size of conidia and microsclerotia among VCGs of C. coccodes are important in the life and disease cycles of this pathogen.

\section{ACKNOWLEDGMENTS}

Funding for this research was obtained from the Minnesota Area II Potato Growers Association and from the USDA-ARS, agreement number 59-0790-4-067. We thank D. Peterson, D. Serfling, R. Nilles, and R. Sherman for technical assistance.

\section{LITERATURE CITED}

1. Andrivon, D., Lucas, J. M., Guerin, C., and Jouan, B. 1998. Colonization of roots, stolons, tubers, and stems of various potato (Solanum tuberosum) cultivars by black dot fungus Colletotrichum coccodes. Plant Pathol. 47:440-445.

2. Barkdoll, A. W., and Davis, J. R. 1992. Distribution of Colletotrichum coccodes in Idaho and variation in pathogenicity on potato. Plant Dis. 76:131-135.

3. Browning, M., Rowley, L. V., Zeng, P., Chandlee, J. M., and Jackson, N. 1999. Morphological, pathogenic, and genetic comparisons of Colletotrichum graminicola isolates from poaceae. Plant Dis. 83:286-292.

4. Burgess, D. R., Porter, I. J., and Parbery, D. G. 1995. Relationship between sunflower development and the onset of stem rot induced by Sclerotinia minor. Aust. J. Exp. Agric. 35:87-92.

5. Campbell, C. L., and Madden, L. V. 1990. Introduction to Plant Disease Epidemiology. John Wiley \& Sons, New York.

6. Christ, B. J. 1991. Effect of disease assessment method on ranking potato cultivars for resistance to early blight. Plant Dis. 75:353-356.

7. Davis, J. R., Mohan, S. K., Sorensen, L. H., and Schneider, A. T. 1988. Colletotrichum coccodes on potato foliage, the association with metribuzin, yield losses, and colonization of tuber. Am. Potato J. 65:475476.

8. Davis, J. R., and Johnson, D. A. 2001. Black dot. Pages 16-18 in: Compendium of Potato Diseases. 2nd ed. W. R. Stevenson, R. Loria, G. D. Franc, and D. P. Weingartner, eds. American Phytopathological Society, St. Paul, MN.

9. Dillard, H. R. 1989. Effect of temperature, wetness duration, and inoculum density on infection and lesion development of Colletotrichum coccodes on tomato fruit. Phytopathology 79:1063-1066.

10. Dita-Rodriguez, M. A., Brommonschenkel, S. H., Matsuoka, K., and Mizubutt, E. S. G. 2006. Components of resistance to early blight in four potato cultivars: Effect of leaf position. J. Phytopathol. 154:230-235.
11. Farley, J. D. 1972. A selective medium for assay of Colletotrichum coccodes in soil. Phytopathology 62:1288-1293.

12. Farley, J. D. 1976. Survival of Colletotrichum coccodes in soil. Phytopathology 66:640-641.

13. Gebhardt, C., Blomendahl, C., Schachtschabel, U., Debener, T., Salamini, F., and Ritter, E. 1989. Identification of $2 n$ breeding lines and $4 n$ varieties of potato ( $S$. tuberosum spp. tuberosum) with RFLP fingerprints. Theor. Appl. Genet. 78:16-22.

14. Hamalainen, J. H, Watanabe, K. N., Valkonen, J. P. T., Arihara, A., Plaisted, R. L., Pehu, E., Miller, L., and Slack, S. A. 1997. Mapping and marker-assisted selection for a gene for extreme resistance to potato virus Y. Theor. Appl. Genet. 94:192-197.

15. Harvey, I. C., Foley, L. M., and Saville, D. J. 1995. Survival and germination of shallow-buried sclerotia of Sclerotinia sclerotiorum in pastures in Canterbury. New Zeal. J. Agric. Res. 38:279-284.

16. Heilmann, L. J., Nitzan, N., Johnson, D. A., Pasche, J. S., Doetkott, C., and Gudmestad, N. C. 2006. Genetic variability in the potato pathogen Colletotrichum coccodes as determined by amplified fragment length polymorphism and vegetative compatibility group analyses. Phytopathology 96:1097-1107.

17. James, W. C. 1981. Estimated losses of crops from plant pathogens. Pages 79-94 in: Handbook of Pest Management in Agriculture. D. Pidmentel, ed. CRC Press, Boca Raton, FL.

18. Joaquim, T. R., and Rowe, R. C. 1990. Reassessment of vegetative compatibility relationships among strain of Verticillium dahliae using nitrate-nonutilizing mutants. Phytopathology 80:1160-1166.

19. Johanson, A., and Thurston, H. D. 1990. The effect of cultivar maturity on the resistance of potatoes to early blight caused by Alternaria solani. Am. Potato J. 67:615-623

20. Johnson, D. A. 1994. Effect of foliar infection caused by Colletotrichum coccodes on yield of Russet Burbank potato. Plant Dis. 78:1075-1078.

21. Johnson, D. A., Carris, L. M., Rogers, J. D. 1997. Morphological and molecular characterization of Colletotrichum nymphaeae and $C$. nupharicola sp. nov. on water-lilies (Nymphaea and Nuphar). Mycol. Res. 101:641-649.

22. Korolev, N., Katan, J., and Katan, T. 2000. Vegetative compatibility groups of Verticillium dahliae in Israel: Their distribution and association with pathogenicity. Phytopathology 90:529-536.

23. Lentner, M. 1993. Experimental Design and Analysis. 2nd ed. Valley Book Company, VA.

24. Leslie, J. F. 1993. Fungal vegetative compatibility. Annu. Rev. Phytopathol. 31:127-150.

25. López-Escudero, F. J., and Blanco-López, M. A. 2005. Isolation and morphologic characterization of Verticillium dahliae isolate from soil. Biotechnology 4:296-304.

26. Miller, P. M. 1955. V8 juice agar as a general purpose medium for fungi and bacteria. Phytopathology 45:461-462

27. Milliken, G. A., and Johnson, D. E. 1992. One-way treatment structure in a completely randomized design with heterogeneious errors. Pages 16-28 in: Analysis of Messy Data, vol 1. Designed Experiments. Chapman and Hall, London, UK.

28. Mordue, J., and Elizabeth, M. 1967. Colletotrichum coccodes. No. 131 in: Descriptions of Pathogenic Fungi and Bacteria. Commonw. Mycol. Inst. Kew, UK.

29. Nitzan, N., Cummings, T. F., and Johnson, D. A. 2005. Effect of seedtuber generation, soilborne inoculum, and azoxystrobin application on development of potato black dot caused by Colletotrichum coccodes. Plant Dis. 89:1181-1185.

30. Nitzan, N., Evans, M., and Johnson, D. A. 2006. Colonization of potato plants after aerial infection by Colletotrichum coccodes, the causal agent of potato black dot. Plant Dis. 90:999-1003.

31. Nitzan, N., Hazanovsky, M., Tal, M., and Tsror, L. 2002. Vegetative compatibility groups in Colletotrichum coccodes, the causal agent of black dot on potato. Phytopathology 92:827-832.

32. Nitzan, N., and Tsror (Lahkim), L. 2003. Effect of temperature and pH on in vitro growth rate and sclerotial density of Colletotrichum coccodes isolates from different VCGs. Am. J. Potato Res. 80:335-339.

33. Nitzan, N., Tsror (Lahkim), L., and Johnson, D. A. 2006. Vegetative compatibility groups and aggressiveness of North American isolates of Colletotrichum coccodes, the causal agent of potato black dot. Plant Dis. 90:1287-1292.

34. Panaccione, D. G., Vaillancourt, L. J., and Hanau, R. M. 1989. Conidial dimorphism in Colletotrichum graminicola. Mycologia 81:876-883.

35. Pelletier, J. R., and Fry, W. E. 1989. Characterization of resistance to early blight in three potato cultivars: Incubation period, lesion expansion rate, and spore production. Phytopathology 79:511-517.

36. Sato, T., Muta, T., Imamura, Y., Nojima, H., Moriwaki, J., and Yaguchi, Y. 2005. Anthracnose of Japanese radish caused by Colletotrichum dematium. J. Gen. Plant Pathol. 71:380-383.

37. Schafer-Pregl, R., Ritter, E., Concilio, L., Hesselbach, J., Lovatti, L., 
Welkemeier, B., Thelen, H., Salamini, F., and Gebhardt, C. 1998. Analysis of quantitative trait loci (QTLs) and quantitative trait alleles (QTAs) for potato tuber yield and starch content. Theor. Appl. Genet. 97:834-846.

38. Shcolnick, S., Dinoor, A., and Tsror (Lahkim), L. 2007. Additional vegetative compatibility groups in Colletotrichum coccodes subpopulations from Europe and Israel. Plant Dis. 91:805-808.

39. Smith, D. 2005. Maintenance of fungi. Pages 83-107 in: Maintenance of Microorganisms. B. E. Kirsop, and J. J. S. Snell, eds. Academic Press, London.

40. Sutton, B. C. 1992. The genus Glomerella and its anamorph Colletotrichum. Pages 1-26 in: Colletotrichum: Biology, Pathology and Control. J. A. Bailey and M. J. Jeger, eds. CAB International, Wallingford, UK.

41. Tsror (Lahkim), L., Erlich, O., and Hazanovsky, M. 1999. Effect of Colletotrichum coccodes on potato yield, tuber quality, and stem colonization during spring and autumn. Plant Dis. 83:561-565.

42. Tu, J. C. 1980. The ontogeny of the sclerotia of Colletotrichum coccodes. Can. J. Bot. 58:631-636.

43. van Eck, H. J., Jacobs, J. E. M., Stam, P., Ton, J., Stiekema, W. J., and Jacobs, E. 1994. Multiple alleles for tuber shape in diploid potato detected by qualitative and quantitative genetic analysis using RFLPs. Genetics 137:303-309.

44. van Eck, H. J., Jacobs, J. E. M., van Dyk, J., Stiekema, W. J., and Jacobsen, E. 1993. Identification and mapping of three flower colour loci of potato ( $S$. tuberosum L.) by RFLP analysis. Theor. Appl. Genet. 86:295-300.

45. Weeks, R. J., and Padhye, A. A. 1982. A mounting media for permanent preparations of microfungi. Mykosen 25:702-704.

46. Yu, X., Hallet, S. G., Sheppard, J., and Watson, A. K. 1997. Application of the placket-Burman experimental design to evaluate nutritional requirement for the production of Colletotrichum coccodes spores. Appl. Microbiol. Biot. 47:301-305.

47. Yu, X., Hallet, S. G., Sheppard, J., and Watson, A. K. 1998. Effects of carbon concentration and carbon-to-nitrogen ratio on growth, conidiation, spore germination and efficacy of the potential bioherbicide Colletotrichum coccodes. J. Ind. Microbiol. Biot. 20:333-338.

48. Zeise, K., and Von Tiedemann, A. 2001. Morphology and physiology differentiation among vegetative compatibility groups of Verticillium dahliae in relation to V. longisporum. J. Phytopathol. 149:469-475. 